If a pure menorrhagia commonly due to :-

(i) Endocrine disturbance.

(ii) Pelvic infections.

(iii) Fibroids or other simple tumours.

(iv) Retroversion.

If independent of the periods.

Under 35 suspect polypus.

Over 35 suspect uterine cancer.

In conclusion attention must again be drawn to the fact that slight irregular bleeding is the only early symptom of uterine cancer. It is, therefore, of the greatest importance, not only that every patient presenting this symptom should be thoroughly examined, but no less so that careful inquiry as to the presence or absence of such irregular bleeding should be made.

\title{
SOME AFFECTIONS OF THE SKIN IN CHILDHOOD.
}

\section{BY GODFREY W. BAMBER, B.A., M.D., M.R.C.P., \\ Physician to the Skin Department, Victoria Hospital for Children, Miller General Hospital and Metropolitan Hospital.}

THE general practitioner with a limited knowledge of skin diseases, who is astonished and confused hy the number of names and descriptions which he finds on consulting a textbook of dermatology, will be relieved to hear that in children the variety of common skin complaints is less than in adults, and that once the correct diagnosis has been made almost all of these conditions are amenable to treatment.

It is convenient to deal with congenital abnormalities first, because children are brought in earliest infancy to see the doctor on account of a very common congenital malformation of the skin, a nævus, or to adopt dermatological nomenclature, an hæmangioma. This developmental defect may appear either as the "strawberry mark" with a slightly raised, irregular, bright red surface, or as a flat patch, the "port wine stain " in which the intensity of the colour depends on the number and depth of the dilated capillaries, or lastly as the subcutaneous hæmangioma, a spongelike tumour from which the blood can easily be expressed. An angioma may conform to one or may be made up of all three types. Parents and relatives are apt to view these marks with apprehension, although there is no necessity for alarm; and consciously or unconsciously influenced by the fear that something must be done urgently, the doctor carries out treatment which in many cases is far too precipitate and vigorous. Angiomata, apart from the port-wine stain, are rarely found in adults, nor do many of these show scars resulting from excessive treatment or even excision. The natural history of angiomata is that they are present at or appear soon after birth ; their rate of growth is for a time more rapid than that of the baby ; then they involute slowly, and often disappear without a trace of their former presence. A good illustration of this is seen on the neck. Every third baby has a flat capillary angioma below the occiput. The colour varies from pale pink to dark red. In a few months most of these marks have gone, although a few, generally the darker ones, may be found when examining the hair of adults. These marks have been said to result from intra-uterine trauma, and 
there is no doubt that angiomata occasionally appear on those areas to which forceps have been applied. Some cases, where the distribution is over the area supplied by a branch of the trigeminal nerve, are associated with hæmangiomata of the meninges, which may give rise to no symptoms until calcification occurs. The strawberry mark, unless it is growing very rapidly or is in an awkward place, such as on the eyelid or lip, or around the auditory meatus, where an increase in size would cause inconvenience, is best left alone. Should interference be considered necessary, the best treatment is to freeze with carbon dioxide snow sufficiently to cause an inflammatory reaction in the endothelial lining of the blood-spaces, with subsequent thrombosis, but not so intensively as to cause necrosis of the superficial tissues. Despite the vascularity of these angiomata, I have never seen serious hæmorrhage from necrotic and infected ulcerated areas. In the subcutaneous cavernous type, sclerosis is best attained by injection; formerly boiling water was used, but its place has now been taken by those substances found useful in the injection treatment of varicose veins. After the first application of treatment do not rush in the following week because the lesion has not gone; the 8 process of fibrosis takes time, so any subsequent treatment, whether by freezing or by injection, should not be undertaken at intervals of less than a month, and not even then 9 if there is a slow but steady diminution in colour or size. The port-wine stain is not so easily dealt with. Freezing is useless unless it is applied long enough to cause destruction of the dermis, which is followed by scarring, which is cosmetically undesirable in many places. To babies I do nothing; to older children who can sit still the application of a Quartz rod attached to a Kromayer lamp gives good results; the areas become paler, but do not disappear completely in many cases. Treatment by excision: is mentioned merely in condemnation; first it leaves an unnecessary scar, secondly the possibility of the development of a keloid cannot be discounted, and lastly, unless the area of excision is wide, a temporary recurrence of the angioma around the scar is not uncommon. Treatment by radium gives rapid and cosmetically satisfactory results. However, it must not be forgotten that this agent exerts its greatest effect on young, rapidly growing tissue, so that its action is much less selective in a baby with an angioma than in an adult with a sarcoma. The possible later development of a radium dermatitis, the unsightliness of which is as great as that of an angioma and is the least serious aspect of this injury, must make one cautious in advocating radium treatment. When treating nævi one should adopt the motto, "Festina lente."

In later childhood the so-called spider nævus, or stellate telangiectasis, is frequently noticed on the face and hands. There is a bright red point where the central vessel emerges from below surrounded by radiating capillaries. If the central vessel is destroyed either by electrolysis or by the galvanocautery, the other capillaries disappear.

Eczema is less common in children than in adults, and its chief incidence is in the No earliest years of life, when it is nearly always of one definite type-the infantile facial eczema. This condition usually starts during the winter months with paroxysmal attacks of itching on the cheeks or forehead. These attacks often occur after a feed, or when the child is brought into a warm room, and cause him to rub violently. Soon the prominent parts of the face show vesiculation and become moist, the exudation drying to form crusts. The eczema nearly always spreads to the scalp, and as a result of rubbing against the pillow the hair in the occipital region becomes thinned, and this area may become almost bare. There may be a diffuse miliary eruption' on the trunk, or discrete 
eczematous patches on the neck, shoulders and limbs. In older infants and young children there is less exudation and crusting; the skin becomes roughened, thickened and shiny with an exaggeration of the normal lines of the skin. After a week or two the eczema begins to subside and then recurs. This alternation persists throughout the winter and spring, but even in the quiescent periods the skin is not really normal. Infantile eczema is three times more common in boys; it affects both breast and bottlefed babies, and is more prevalent in fat children, the majority of whom are over the normal weight at birth and later. Asthma, eczema and migraine are frequently found in the family history. In these babies asthma is often called bronchitis, the attacks come suddenly and as rapidly go, and the physical signs are out of proportion to the symptoms. Babies who suffer from this form of eczema are said to have the exudative diathesis, a condition characterized by Habbiness, with rapid fluctuations in weight during illness, and by a tendency to catarrhal affections and spasmophilia. The protection of the face is the chief object of external treatment. To prevent rubbing, the arms should be splinted or fastened down to the clothes. At night the arms are controlled either by fastening them to the sides of the cot, or by putting the child up to the neck in a pillow-slip. Except when the moist or excoriated areas have become infected, the best application is 3 per cent. crude coal-tar in zinc paste. This is a dirty application, and it is convenient to cover the face with a mask which also helps to keep the skin at an even temperature. No soap and water is to be used on the affected parts, the old ointment is cleaned off either with olive oil or with medicinal paraffin. If the child is above the normal weight, reduce the diet, especially the fluid portion, and increase the intervals between feeds to four hours. These babies are often greedy feeders who overdistend and disorder their stomachs. Sometimes the primary itching is due to urticaria, and an insufficient breaking down of the food proteins, and the entry into the blood-stream of more complex particles is considered by some people to be the cause of this condition. Drugs are not indicated, although some children do well on acid milk.

With the return of warmer weather most babies lose their eczema, but a few develop itchy patches in the bends of the elbows and behind the knees with ensuing lichenification of the skin. This flexural pruritus or Besnier's prurigo may not appear until some years later, and often persists until puberty. It is more definitely associated with asthma than is facial eczema, of which there is nearly always a history. In some children the attacks of itching in the flexures are more common at one season than at another, most of them become free at the seaside, and the attacks of asthma may coincide or alternate with the dermal exacerbations. Both conditions are probably manifestations of allergy, and desensitization should be tried. Occasionally a specific antigen is found, but generally non-specific methods of desensitization have to be used in treatment. The external treatment is the same as that used for facial eczema. Secondary infection of excoriations must be dealt with before using tar paste.

All who have to deal with children are familiar with papular urticaria or lichen urticatus. The child comes out in crops of small, red, itchy spots, each of which has a tiny central vesicle. In a few hours the erythema fades leaving the vesicle surmounting a small papule. Frequently the vesicle is dug out by the finger-nails and is replaced by a blood crust, and, as might be expected, secondary infection is not uncommon, giving rise to lesions of the impetign type. A less frequent clinical form is one in which the 
papules are more or less absent, the vesicles are larger and may be blister-like, especially in the region of the legs and feet. This type is more frequent in small children and may be confused with the bullous type of impetigo, but in the absence of the more usual papules elsewhere, the itchiness and symmetry of the eruption will indicate the diagnosis. The papular form, on account of the itching at night, may be confused with scabies, but the absence of burrows and the typical distribution excludes this complaint. It is very rare for the vesicular distribution to be uniform and widespread so as to give rise to suspicions of varicella. It can be said that when such a vesicular eruption appears in a child already in hospital, the case is almost certainly one of varicella, because even the most persistent cases of papular urticaria cease when the child is admitted. Hallam, who has thoroughly investigated many cases, says that the complaint may be controlled by detaining the child in hospital for the night only. The ætiology is still unknown. The incidence is much greater in the summer and autumn, although in a few children it persists throughout the year. It is higher in the hospital class of patient, and Hallam believes that the exciting cause is probably associated with the home sleeping arrangements. It is curious that attacks may be affected by locality. A first attack may occur on moving to a new district, and outbreaks may cease under the same conditions. In some cases the exciting cause is something in the diet, possibly a protein, in other cases a limitation of the carbohydrate intake has been found useful. It has been surmised that there occurs a temporary derangement of the liver which allows foreign protein to enter the general circulation. Small doses of rhubarb and soda before meals will be found extremely useful in the majority of cases. To diminish the locad irritation, dab on I per cent. phenol in calamine lotion. The prognosis is good; the children grow out of the complaint, and if the lesions are not secondarily infected ne scarring occurs. In some children there is an excess of pigment formed in the areas formerly occupied by the papules, which persists for some time. When this is present it does not make the case one of urticaria pigmentosa.

Impetigo contagiosa is the commonest infective affection of the skin in children, and may be primary or secondary to some other condition which leads to itching and scratching, such as scabies, urticaria papulosa, and pediculosis capitis. Impetigo as a rule clears up fairly rapidly, and this combined with the frequency with which it occurs has led to a more or less stereotyped mode of treatment. The recognition of the disease appears in many cases to set off a conditioned reflex, the unvarying response being the inspiration, ammoniated mercury ointment, and a box of this is handed out without further thought or advice. The primary lesion is a vesicle just below the horny layer. Should the vesicle rupture, serum oozes out and coagulates to form the usual type of crust which has a stuck-on appearance. When the horny layer is very thick as in the palm, or if the adhesion between the horny layer and the next is less than normal, the vesicle does not burst but increases in size to form a blister, which may burst later, exposing a bright red surface with a border of epithelium. Several of these ring-like areas may run together forming a patch with an arcuate outline. In the treatment of impetigo it is necessary that all crusts should be removed each time any antiseptic is applied. This is not always sufficiently stressed to the child's mother, who may neglect to bathe off the crusts either because the operation is slightly painful, or because there might be a slight oozing of blood. The next point is that mercury ointment is messy; its base has a low melting point, so that the ointment does not stay on the site of 
application, but runs down to a lower place. As an antiseptic ointment use something with a stiffer base, such as the one introduced by Whitfield, 3 per cent. yellow oxide of mercury in zinc paste. The only place where the ointment is preferable to the paste is on the scalp, and when impetigo occurs here the presence of pediculosis capitis should always be suspected and sought. The bullous type of impetigo responds more quickly to a lotion such as $I$ in $I, 000$ flavine in normal saline.

There is a type of impetiginous infection to which special attention must be drawn, because it is recurrent, it involves more of the skin in successive attacks, and if not adequately treated leaves the patient when an adolescent or young adult with a chronic infective dermatitis involving the scalp, the hairy parts of the axillæ and pubis, the skin around the ears, the eyes, and in the folds of the body, including the under-surface of the mammæ, with occasional outbreaks on other parts of the body. This unenviable and intractable state nearly always begins in childhood with cracks behind and below the auricles. Sometimes crusts form over these cracks, but more often the lesions resemble the bullous type of impetigo seen elsewhere. The lesions when they first occur may clear up rapidly under treatment, but a small crack may remain, and a subsequent outbreak spreads further involving the auricle and the mastoid region together with the adjacent hair margin. With successive attacks the scalp is affected in ever-widening circles around the ears. Between the attacks the scalp in these areas is covered with fine dry scales. Finally, there is an acute attack involving the entire scalp which is covered with a gummy serum with patches of pustulation and crusting. Sabouraud says that with proper care and attention a cure can be effected in two years. Unfortunately the majority of children with this infection belong to the poorer class of hospital patient, and owing to the unhygienic home environment and the lack of hospital beds for their treatment they progress much more slowly. It is apparent that no effort should be spared from the onset to prevent the gradual lowering of the resistance of the skin. Cracks which occur around the ears, less frequently around the nostrils and the corners of the mouth, should be carefully and thoroughly treated by the methods used for impetigo elsewhere. Emulsio flavine, an oily solution, will be found very useful in this region. When the obvious infective element has subsided, the application of tinct. benzoini co. will generally cause persistent cracks to heal. When dealing with an acute outbreak on the scalp avoid ointments which serve only to aggravate the dermatitis : the best application is lead lotion. In a few days the inflammatory reaction will have diminished sufficiently for a mild antiseptic lotion of flavine or resorcin to be tolerated. The next step is to incorporate some oil into the lotion, but should the scalp begin to show signs of intolerance an earlier stage of treatment must be resorted to again. Particular attention should be paid to the child's general health and hygiene; there should be an adequate and well-balanced diet, while tonics, extra vitamins and carbon arc light baths may be given.

Ringworm infections which are important, not because they are particularly harmful to the child, but because of the extreme care which has to be taken to prevent dissemination, now appear to be diminishing. More than 90 per cent. of ringworm infections of the scalp are due to the microsporon, and it is noteworthy that this type of fungus very rarely infests the hair of those who have reached puberty. Clinically there are one or more circular patches covered with dirty white scales, and the hairs in the patches are broken off fairly uniformly about $\frac{1}{8} \mathrm{in}$. above the level of the scalp. These 
stumps, being surrounded by a sheath of spores, appear to be coated with a greyish powder. If the scalp is examined under Wood's light in a dark room the infected hairs fluoresce. Not infrequently on examination under the lamp small groups of infected hairs are seen to be dotted over the scalp, sometimes there may be only three or four hairs in one spot, and these are liable to be overlooked in the routine clinical examination. In microsporon, ectothrix, favus, but not in endothrix infections, the hairs fluoresce, and this property is useful not only in diagnosis, but also after treatment to see that the scalp is free from infected hairs, so that the case can be discharged. Different clinical appearances are seen in ectothrix and endothrix trichophyton infections of the hair. In endothrix infections the scurf is less abundant, and stumps are fewer and are not so easily found among the longer hairs. Only in the very rare instances when the infection is due to the trichophyton acuminatum is there any clinical appearance resembling alopecia areata. Then the affected area is bald, but the mouths of the hair follicles appear as black dots because the affected hairs have broken off at that level. The ectothrix, the normal habitat of which is on animals, causes a suppurative reaction. The infected areas become boggy swellings with points of pus around the mouths of the follicles from which the infected hairs can easily be extracted. This form of lesion is the kerion celsi. On the smooth skin the appearance of the lesion varies with the type of infecting fungus. The microsporon causes uniform, red, scaly, discoid areas, usually not larger than the finger-nail, which are seen most often on the neck and face when the scalp is infected. The endothrix gives rise to scaly patches with a vesicular margin which extends peripherally while the middle heals, but this part may be reinfected, so 8 that the lesion is formed of concentric rings. Ectothrix lesions are more inflammatory and appear as raised plaques often with pustulation. Whenever I see a child with? pityriasis rosea this condition has almost always been previously called ringworm. The herald patch when present usually appears on the covered parts, an unlikely place for ringworm to reach first, while the full eruption appearing almost simultaneously and symmetrically over the trunk and the proximal part of the limbs and sparing the face, together with the distal part of the limbs, the most likely sites of contact with the fungus, makes the suggestion of ringworm most improbable and a thoughtless guess. Patches of ringworm on the smooth skin are easily and simply treated by rubbing in Whitfield's ointment. In the kerion suppuration is a natural attempt to cure by making the hairs loose. This can be aided by pulling out the loose affected hairs and by the application of hot fomentations. The infection of the shaft of the hair extends too deeply for the effective penetration of any antiparasitic remedy, so that the only certain way of curing a microsporon or endothrix infection is to epilate. Manual epilation alone is useless, because the infected and structually weakened hair, if pulled, breaks above the lowest level to which the fungus penetrates, a short distance above the bulb. The old method of treatment was to rub in an irritating ointment until suppuration ensued, so that clinically the case became like an ectothrix one. The modern way is to epilate by X-rays or the less satisfactory thallium acetate. The hairs fall out three weeks after the application of X-rays, and in the interval Whitfield's ointment is applied to kill off any infection in the scales. Although the scalp is not adequately covered with the new hair until about three months later, the child is no longer a source of infection and can return to school after all the old hairs have been shed.

In scabies the places which should be examined for burrows are the interdigital 
clefts, wrists, elbows, anterior axillary folds, buttocks, and popliteal spaces. When examining children do not forget to look at the sides and soles of the feet, because burrows are frequently seen more easily here than in other sites. In very small children, especially infants, the infestation frequently passes undiagnosed because of the polymorphy of the eruption ; the presence of patches of eczema and urticaria along with vesicles, bullæ, pustules, and excoriations is likely to mislead those who are not familiar with the clinical aspect of infantile scabies. The mother should always be examined. When an infected child has been sleeping with another one, this latter, even if it shows no definite signs of scabies, should also be treated, because if not, definite signs will almost certainly appear later, and the first child may be reinfected. Sulphur ointment is commonly used for the treatment of scabies. After a good soaking in a warm bath where all the burrows have been opened by rubbing or by scrubbing, the child is covered in ointment from the toes to the neck, applications being made on three successive nights. After this time further sulphur applications should not be used. It must not be forgotten that for some skins sulphur is an irritant and may at the end of three days set up a certain amount of itching. . Under a mistaken belief that the scabies is not eradicated the treatment may be continued so that a sulphur dermatitis occurs. For small children the percentage of sulphur is reduced to half, and for infants balsam of peru is substituted. About $\mathbf{I}_{3}^{2} \mathrm{Oz}$. of ointment are necessary for a single application in an adult, so the amount for a child can be guessed fairly easily.

To get rid of pediculosis capitis soak the hair in a warm carbolic lotion (dilution $I$ in 40) and wrap up the head in a towel for an hour. Afterwards the nits are combed out with a fine metal comb. In the L.C.C. clinics children with dirty heads are treated by rubbing in tar oil compound for ten minutes. This substance is 5 per cent. tar oil in an inert oily base. The head is then shampooed and afterwards combed with a Secker comb.

Rashes in the napkin area are common in infants. It is usual to find the napkin enclosed in indiarubber drawers which are constricted around the waist and thighs. These articles, while excellent for the prevention of the soiling of clothes, yet because of the lack of ventilation keep the enclosed area in a hot moist atmosphere, and I believe them to be a predisposing and aggravating factor in some eruptions in this area. Not merely babies who have suffered from thrush, but others also get an eruption in the napkin area due to a monilia infection. The rash consists of discoid areas from which the surface epithelium has been lost. These areas are bordered by a fringe of undermined epidermis. By-peripheral extension the patches become confluent and usually involve the perineum and groins. Occasionally the eruption may spread much farther, the fresh outlying lesions being either superficial vesicles or slightly scaly red macules. The application of an antiseptic such as flavine in linimentum calamine clears up the infection fairly rapidly. A more common eruption is the so-called napkin dermatitis. In these cases the mother will frequently volunteer the information that the napkins smell strongly, and the pungent odour of ammonia may be recognized when the baby is being undressed for examination. The ammonia is a decomposition product of urine and is due to the action of a bacillus which is more abundant in alkaline stools. Hence the incidence of the eruption is greater in bottle-fed babies. Most of the ammonia formed remains in solution, so the irritant action occurs on those places which come in contact with the wet napkin, e.g., the buttocks, perineum, genitals, also the calves and 
occasionally the heels. There may be several forms of rash, the commonest is an erythema. Less frequently there is a number of large dull red papules on the prominent parts; sometimes the surfaces of the papules are eroded giving an appearance like condylomata. In congenital syphilis, however, the papules appear more on the flexor aspect and not on the most prominent parts, the skin between the syphilitic papules is more normal, and there are usually characteristic lesions elsewhere, e.g., on the face, palms and soles. The treatment of napkin dermatitis is simple. Decomposition of the urine is prevented by dipping the washed napkins in a $I$ in 5,000 solution of hydrarg. perchlor. before they are put to dry. Linimentum calamine is applied to the affected parts each time the napkin is changed.

Warts are more common in children than in adults. There is one type which is frequently diagnosed erroneously as a corn. This is the plantar wart which may appear either singly or in small groups at points of pressure on the foot. The lesion appears as a narrow ring of epidermis often differing slightly in colour from the rest of the horny layer enclosing an area of fissured epithelium which is extremely tender when pressed upon and which may make walking painful. When considering the treatment of warts the fact that they disappear spontaneously after a shorter or longer interval must not be forgotten, so any method which may leave unsightly scars on the face should be avoided. The pain arising from a plantar wart makes treatment imperative. The most satisfactory for this is an application of X-rays. After a suitable dose the pain disappears in a few days, although the lesion itself takes about a month to disappear. X-rays are also suitable for large isolated warts and for those involving the nail-fold and the nail-bed. In general practice some caustic application is the most convenient for ordinary warts The most certain of these is trichloracetic acid, although great care has to be taken to avoid any spread to adjacent normal skin. When plane warts are few in number they can be destroyed by the galvanocautery; when numerous it is better to apply a spirit lotion containing perchloride of mercury or resorcin. Several internal remedies have been suggested for warts but I have found none of them certain in action.

\title{
THE TREATMENT OF CHRONIC B. COLI INFECTIONS OF THE URINARY TRACT BY A KOTOGENIC DIET.
}

\author{
BY C. M. WILSON, M.D., F.R.C.P., \\ Senior Physician to Out-patients, and Dean of the Medical School, St. Mary's Hospital.
}

IN May of last year Professor Cabot was kind enough to give me particulars of the ketogenic diet, which as a result of the research work of Clarke and Helmholtz was in use at the Mayo Institute, for infections of the urinary tract by $B$. coli. I myself had for some months been making quite unsuccessful attempts to get rid of this particular infection, and I began the diet with hopes chastened by many previous failures. On the sixth day my urine was sterile: in the eight months which have elapsed since that date there has been no recurrence. My experience of the diet during that time has been confined to chronic cases, and in the main to adults. The results have been remarkable. I do not propose to record them here, because I believe it will be more helpful to dwell 